\title{
Anxiety disorders in children and adolescents
}

\section{Article}

\section{Accepted Version}

Creative Commons: Attribution-Noncommercial-No Derivative Works 4.0

Hill, C., Waite, P. and Creswell, C. (2016) Anxiety disorders in children and adolescents. Paediatrics and Child Health, 26 (12). pp. 548-553. ISSN 1751-7222 doi:

https://doi.org/10.1016/j.paed.2016.08.007 Available at https://centaur.reading.ac.uk/66854/

It is advisable to refer to the publisher's version if you intend to cite from the work. See Guidance on citing.

To link to this article DOI: http://dx.doi.org/10.1016/j.paed.2016.08.007

Publisher: Elsevier

All outputs in CentAUR are protected by Intellectual Property Rights law, including copyright law. Copyright and IPR is retained by the creators or other copyright holders. Terms and conditions for use of this material are defined in the End User Agreement.

\section{www.reading.ac.uk/centaur}

\section{CentAUR}

Central Archive at the University of Reading

Reading's research outputs online 


\section{Anxiety disorders in children and adolescents}

Claire Hill, Polly Waite, Cathy Creswell

Claire Hill, BSc, PhD, DClinPsy. Clinical Postdoctoral Research Fellow \& Hon.

Clinical Psychologist

School of Psychology \& Clinical Language Sciences, University of Reading,

Whiteknights, Reading, RG6 6AL. Tel: +44 (0)118 378 8523. Fax: +44 (0)118 378

6715

claire.hill@reading.ac.uk

Polly Waite, BSc, PhD, DClinPsy. Clinical Lecturer \& Hon. Clinical Psychologist

School of Psychology \& Clinical Language Sciences, University of Reading,

Whiteknights, Reading, RG6 6AL. Tel: +44 (0)118 378 8523. Fax: +44 (0)118 378

6715

p.l.waite@reading.ac.uk

Cathy Creswell, BA Ox(Hons), PhD, DClinPsy. Professor of Developmental Clinical Psychology \& Hon. Consultant Clinical Psychologist

School of Psychology \& Clinical Language Sciences, University of Reading,

Whiteknights, Reading, RG6 6AL. Tel: +44 (0)118 378 8523. Fax: +44 (0)118 378

6715

c.creswell@reading.ac.uk

Corresponding Author: 
Claire Hill, School of Psychology \& Clinical Language Sciences, University of Reading, Whiteknights, Reading, RG66AL. claire.hill@reading.ac.uk 


\section{Abstract}

Anxiety disorders are among the most common psychiatric disorders in children and adolescents. They commonly interfere with peer relationships, schooling and family life, and persist into adulthood if left untreated. This paper gives an overview of the identification, assessment and treatment of anxiety disorders in youth. Identification of anxiety disorders is often poor and many young people with anxiety disorders go untreated. We present a brief review of the evidence base for pharmacological and psychological treatment approaches to the management of anxiety disorders in youth. Both have been found to be effective in the treatment of anxiety disorders, although psychological treatments such as Cognitive Behavioural Therapy (CBT) are considered the first line treatment due to relative benefits in terms of patient safety and parental preference. Low intensity CBT approaches such as bibliotherapy and online therapies are effective and have the potential to improve access to evidence based interventions. CBT approaches have also been found to be effective with particular patient groups, such as those with long-term physical health conditions and autism spectrum disorders, who are at an increased risk of anxiety disorders.

\section{Keywords:}

anxiety; child; adolescent; cognitive behavioural therapy; SSRIs; psychological; pharmacological; assessment; treatment 


\section{Introduction}

Anxiety disorders are among the most common mental health disorders in youth, with prevalence rates between $9 \%$ to $32 \%$ during childhood and adolescence. Lifetime prevalence of anxiety disorders is estimated at $27 \%$, with the average age of onset in childhood.

\section{Classification}

Anxiety disorders are characterised by excessive worries, fears and negative beliefs, avoidance of specific situations or objects, and physical symptoms such as increased heart rate, quicker and shallower breathing, sweating, chest or stomach pains. The diagnostic manual DSM-5 specifies a number of anxiety disorders including Separation Anxiety Disorder, Specific Phobia, Social Anxiety Disorder (previously Social Phobia), Generalised Anxiety Disorder, Panic Disorder, Agoraphobia, and Selective Mutism (see Table 1). "Panic attack" (sudden onset of intense fear with cognitive and physical symptoms such as heart palpitations, breathlessness, dizziness, derealisation and fear of dying) is included as a specifier that can be applied across the DSM-5 disorders, as it is recognised that panic attacks can be implicated in a range of disorders. Unlike previous versions, DSM-5 does not classify Obsessive Compulsive Disorder (OCD) and Post-Traumatic Stress Disorder (PTSD) as Anxiety Disorders (they are now listed under ObsessiveCompulsive and Related Disorders, and Trauma-Related and Stressor-Related Disorders, respectively) and, accordingly, we will not discuss them further here.

Although the specifics of each anxiety disorder vary (see Table 1), they share some common features. These include overestimation of the actual threat and danger 
associated with the feared situation, underestimation of the individual's ability to cope, and significant interference in normal life experiences such as friendships, school and family life.

Impact

Anxiety disorders cause significant interference with the day-to-day functioning of children and young people, affecting school performance, social functioning, family life, leisure activities and sleep. It is also important to note that, if anxiety disorders are left untreated, they tend to run a chronic course and often persist into adulthood. Anxiety disorders typically co-occur, are often comorbid with other disorders such as depression, and predict the development of other serious mental health problems in later life such as depression, substance misuse and risk of suicide. Given how common and damaging childhood anxiety disorders can be, it is important to ensure that these are promptly identified and appropriately managed using evidence-based treatments.

\section{Development and maintenance}

A multitude of factors has been implicated in the development and maintenance of childhood anxiety disorders. Anxiety disorders tend to run in families, with anxious children more likely to have anxious parents than non-anxious children. Furthermore, children of parents with an anxiety disorder are 7 times more likely to have an anxiety disorder themselves, compared to children of parents with no psychiatric disorder. This intergenerational transmission has led researchers to explore genetic links to anxiety disorders, with twin studies showing that approximately $40 \%$ of the variance in anxious symptoms is accounted for by heritability. 
Child temperament is also thought to be an important factor in the development of anxiety disorders. In particular, an inhibited temperament in early childhood is a risk factor for anxiety disorders in middle childhood and mid-adolescence. Other hypothesised vulnerability factors include information processing biases such as interpretation of ambiguous situations as threatening and heightened attention towards potentially threatening environmental cues. Environmental factors have also been implicated, including adverse life events and exposure to negative information or modelling of anxious responses. Recent studies have suggested that anxious children can be particularly attuned to this information. Furthermore, child characteristics can elicit certain parental behaviours such as overprotection, which although well intended and understandable, may serve to maintain anxiety as they may allow the child to avoid anxiety-provoking situations. The main takeaway message from all of this is that the development of anxiety disorders in youth is typically underpinned by a complex interplay of several factors.

\section{Management of anxiety disorders in children and young people}

\section{Seeking help}

Children and adolescents with anxiety problems do not usually seek help from medical professionals themselves. Instead, concerns are more likely to be raised by their parents, for example to their General Practitioner (GP) or other professionals (e.g. school staff). Rates of help seeking for anxiety disorders appear to be lower than for other mental health problems in childhood and the reasons for this are not well understood. Common barriers that prevent parents from seeking help for their 
child in relation to mental health concerns more generally include a lack of knowledge and understanding of mental health problems and the help seeking process, family circumstances (e.g. limited time and competing commitments), negative attitudes towards service providers and treatment, and perceived difficulties with the mental health system (e.g. long waiting times and a lack of appointment flexibility). The specific barriers to help-seeking in the context of child and adolescent anxiety disorders remain unclear.

Identification of potential anxiety disorders in children and young people GPs (alongside Common/Single Point of Entry services) have a gatekeeper role in relation to Child and Adolescent Mental Health Services (CAMHS) and therefore recognition and appropriate management within the GP consultation is key for the timely detection of anxiety disorders and access to effective interventions. Despite this, studies have found that GPs are often poor at recognising mental health problems in young people, especially if parents do not raise concerns. Anxiety can often present as somatic complaints in children (e.g. stomach aches and headaches), and young people may not be as forthcoming as adults in bringing up mental health issues, making anxiety all the more difficult to identify. In addition, GPs report that a lack of confidence, knowledge, time, providers and resources also reduce their ability to detect anxiety and their willingness to refer children and young people for treatment.

Once anxiety is identified as a potential area of concern, assessment of (i) the severity and duration of symptoms and (ii) the associated impairment will enable the GP to determine whether fears and worries are likely to be developmentally 
appropriate or indicate an anxiety disorder. Thus if the level of anxiety is high and disproportionate to the actual threat involved from the feared situation or stimulus, and these difficulties have persisted for several months and cause significant interference in the young person's daily functioning e.g. peer relationships, schoolwork, school attendance or family life, a referral for further assessment by specialist services is indicated. Unfortunately, no brief screening measures for childhood anxiety disorders are currently available. However, there are freely available parent and child-report questionnaire measures, such as the Revised Children's Anxiety and Depression Scale (RCADS; 47 items), which can be reliably used in children aged 7 years and above. The RCADS has subscales that broadly align with the different diagnostic categories and clinical cut-off scores are provided. The RCADS also involves questions about low mood, a common comorbid problem.

\section{Assessment of anxiety disorders in children and young people}

The National Institute of Health and Care Excellence (NICE) guidelines for the assessment and treatment of anxiety disorders have primarily focused on adults. However, the recent NICE guidelines for social anxiety disorder includes recommendations for children and adolescents, and some of the broader principles may be applied to other anxiety disorders. NICE recommends that a comprehensive assessment should be undertaken by an appropriate healthcare professional. The young person should have the opportunity to be interviewed on his or her own, however the parent(s) or carer(s) should also be included in the assessment as they are valuable informants. Because young people with anxiety disorders commonly experience comorbid conditions, in addition to assessing anxiety related symptoms and disturbance, the assessment should also cover other typically co-occurring 
mental health difficulties (e.g. mood disorders) as well as neurodevelopmental conditions, alcohol and substance misuse.

In the UK, CAMHS routinely assess and monitor anxiety and mood symptoms using the RCADS as part of the Children and Young People's Improving Access to Psychological Therapies Programme (CYP IAPT). This is an initiative that began in 2011 which aims to ensure that families receive an evidence-based and outcomefocused service in CAMHS.

The presence of an anxiety disorder (rather than anxiety symptoms) can be assessed using structured clinical interviews, such as the Anxiety Disorders Interview Schedule for children and parents (ADIS-C/P). The current version aligns with DSM-IV criteria and considers whether a child meets criteria for a specific anxiety diagnosis based on symptoms and the associated level of interference with functioning.

\section{Treatment of Anxiety Disorders in Children and Young People}

\section{Accessing treatment}

In the UK, treatment for childhood anxiety disorders is typically accessed through local CAMHS. Although it is not uncommon for children and adolescents to receive input from other services provided by the local authority, charity organisations or school staff, these services may not be subject to the same requirements to deliver evidence-based treatments. 


\section{Evidence-based treatments}

The majority of randomised controlled trials (RCTs) for the treatment of anxiety disorders in children and adolescents have evaluated pharmacotherapy and/or Cognitive Behavioural Therapy (CBT). Research into other approaches such as systemic therapy and psychodynamic therapy is lacking and little is currently known about their efficacy and effectiveness.

\section{Pharmacotherapy}

There is some evidence to suggest that anxiolytic medication is an effective treatment for anxiety disorders in combination with CBT or alone, although none are currently licensed in the UK for use with young people with anxiety disorders. The majority of treatment trials have evaluated selective serotonin reuptake inhibitors (SSRIs), and this is considered to be the first line pharmacotherapy. While recent studies have suggested that SSRIs are generally safe and tolerable, they are associated with more physical adverse events (such as headache, insomnia and sedation) than CBT, especially in preadolescent children. Furthermore, the optimum treatment duration and the clinical consequences of stopping medication have yet to be evaluated. Perhaps in reflection of these limitations, parents tend to report a preference for psychological interventions over medication for the treatment of anxiety disorders in children and young people. As such, the NICE guidelines for social anxiety disorder recommend that CBT is the first-line treatment for anxiety disorders in children and young people and that pharmacological interventions are not routinely prescribed (i.e. they should only be offered within specialist services). Whilst specific guidelines for other anxiety disorders have yet to be published, the literature on which these recommendations were based was not specific to social 
anxiety disorder and as such they can be considered applicable across the range of anxiety disorders that are experienced by children and young people.

\section{Cognitive Behavioural Therapy (CBT)}

CBT is a psychological therapy based on the theory that interconnectivity between thoughts, feelings and behaviours maintains difficulties such as anxiety. For example, when faced with their parent going out for the evening, a child with separation anxiety disorder may think that their parent will get hurt in a car accident, leading to worry and scared feelings, physical symptoms such as agitation and attempts to avoid the situation, for example, by repeatedly asking their parent not to go out. Understandably, both young people and adults alike tend to avoid situations that make them feel anxious, and consequently the anxiety reduces in the shortterm. However, this can prevent the child or adolescent from having the opportunity to learn that (i) the feared outcome may not happen and (ii) they can cope with the challenge. So when a similar situation arises in the future, they feel anxious and the cycle is repeated. CBT looks at how to break unhelpful patterns by focusing on what is maintaining the difficulty, rather than what caused the problem. For example, it may be that the start of the child's separation anxiety could be traced back to a close friend having a car accident, which is helpful in understanding the context of the difficulties they are experiencing, but a specific focus on this is not necessary in order to break the links between the thoughts, feelings and behaviour that are maintaining the difficulties in the here and now.

Due to the high level of comorbidity between anxiety disorders in children and adolescents, CBT has tended to be generic in its approach, rather than focused on a 
specific disorder. Generic CBT approaches have been particularly influenced by the manualised CBT "Coping Cat" programme that was developed by Philip Kendall in the US, and typically involve psychoeducation, identification and modification of anxious thoughts through cognitive restructuring, graded exposure to the feared object or situation, relaxation techniques and problem solving. CBT for anxiety disorders usually involves between 9 to 20 individual face-to-face CBT sessions with the child or adolescent. Although it is not clear which of these components are necessary or sufficient in treatment, exposure to the feared stimulus or situation (i.e. 'facing fears') appears to be particularly important.

Numerous studies have evaluated the effectiveness of CBT for anxiety disorders in children and adolescents. Most of these have compared CBT to a waitlist control group, although a few studies have included an active control condition such as educational support. A recent Cochrane Review concluded that CBT was significantly more effective than a waitlist control for a range of childhood anxiety disorders. Indeed, systematic reviews consistently report that CBT results in recovery from diagnosis of a primary anxiety disorder in approximately $60 \%$ of cases immediately after treatment, and this proportion rises to around $70 \%$ in the following 6 to 12 months. CBT has not consistently been found to be superior to active control interventions, highlighting the fact that ongoing work is needed to establish what the critical ingredients of effective treatments are and to improve treatment outcomes. However, CBT is currently the only psychological therapy that has been shown to be effective in a number of randomised controlled trials, and as such it is recommended as the first-line treatment for childhood anxiety disorders. CBT appears to be 
similarly effective whether delivered individually face to face or within a group, and with and without parental support.

As noted above, approximately $40 \%$ of young people retain their primary anxiety disorder diagnosis after CBT yet it remains unclear why some young people do not respond while others do. Small-scale studies have failed to find consistent predictors of treatment outcome, however an international multisite and multi-trial study of 1519 children and young people recently concluded that a diagnosis of social anxiety disorder, presence of a comorbid externalising or internalising disorder, and parental psychopathology predicted poorer treatment outcomes. Age, gender and treatment type (individual, group, self-help) did not predict response to CBT. Some approaches to improve outcomes for young people with a relatively poor prognosis include CBT programmes that incorporate social skills training and disorder-specific Cognitive Therapy for children and adolescents (respectively) with social anxiety disorder, although larger studies are needed.

Treatment trials have typically evaluated CBT amongst children and adolescents covering broad age ranges, usually from about 7 years, and similar treatments are used across the age range (with some adaptations to make them more 'teenfriendly'). Although a recent meta-analysis concluded that adolescents showed comparable benefits to younger children after CBT, a subsequent large treatment trial found that adolescents with anxiety disorders had significantly poorer treatment outcomes, than anxious children. There are a number of reasons why this may be the case, including that adolescents tend to have more severe symptoms, greater comorbidity with mood disorders and more frequent school refusal than children 
presenting with anxiety disorders, all factors that have been linked to relatively poor treatment outcomes. Nonetheless, some treatments that were originally designed for adults have recently been successfully adapted for use with adolescents and in the case of social anxiety disorder this has had extremely promising results.

\section{Low intensity treatments}

Low intensity treatments involve providing the least possible therapeutic input possible while maintaining treatment efficacy, and are designed to be used within a stepped model of care delivery, where more intensive treatments are reserved for those who require greater input or do not respond to lower intensity treatments. CBT can be successfully delivered in less intense forms such as through guided selfhelp/bibliotherapy (providing written materials to parents to enable them to use CBT strategies with their child with therapist support) and via computer-based programmes (CCBT) or online ("e-therapies"). Numerous studies have shown that these ways of delivering treatment can produce similar results to more intensive individual child treatments or face-to-face therapies, although few studies have made this direct comparison.

For preadolescent children, using written materials to work through parents rather than directly with the child brings many benefits such as the ability for parents to implement the strategies beyond the therapy sessions and as and when anxieties emerge after therapy ceases. In addition, children do not have to miss school or activities to attend therapy, and it prevents the child from experiencing stigma associated with attending therapy. In the UK, we have shown that a brief ( 5 and a half hours) therapist-guided self-help programme delivered to parents of children 
aged 7-12 years old is effective in reducing the number of children who have an anxiety disorder, with similar outcomes to those typically achieved with 12-16 hours of individual child CBT. Furthermore, treatment outcomes did not differ in those supported by a "novice" CBT therapist (e.g. psychology graduates) compared to an experienced clinician (e.g. a Clinical Psychologist), supporting the use of this approach within a stepped care model of treatment delivery.

Delivering psychological treatments online ("e-therapies") also has the potential to increase access to evidence-based treatments and improve the efficiency and costeffectiveness of services. A plethora of mental health related smartphone/tablet applications ("apps") are available, although we are not aware of any specifically aimed at treating anxiety disorders in youth that have an evidence-base. Platforms for accessing therapy online (e.g. Kooth.com) are commissioned within some NHS Trusts, however it is yet to be demonstrated whether accessing psychological therapies online in this manner is effective for treating childhood anxiety disorders. However, a handful of online (e.g. BRAVE-online) or computer-based (e.g. Camp Cope-A-Lot) treatments specifically for anxiety disorders in children and adolescents have been developed and evaluated in randomised controlled trials, predominantly within Australia and the US. Studies consistently indicate that these produce similar outcomes to face-to-face treatments (though again direct comparisons are sparse) in reducing the number of children and adolescents who meet criteria for an anxiety diagnosis. Whilst encouraging, these interventions often struggle to make it past the arena of academic evaluation, and dissemination into the NHS is rare. Despite this, service users have an appetite for accessing therapy online and there is growing recognition that academics, clinicians and industry need to work together to develop, 
evaluate and disseminate e-therapies. NICE is currently working on guidelines relating to the level of evidence needed for the endorsement of mental health apps and online treatments, which should bring clarity and guidance to the commissioning of e-therapies.

\section{Early intervention/prevention}

There is a clear argument for intervening early with anxiety disorders due to their chronic nature. Selective interventions that target young children with known risk factors for anxiety disorders have started to emerge. In Australia, an intervention targeted at pre-schoolers with an inhibited temperament (i.e. behaviourally inhibited, socially withdrawn, shy) successfully changed the expected trajectory of this at-risk group and lowered the frequency and severity of anxiety disorders that developed during a 3-year period compared to yearly monitoring. Such selective interventions show promise but have yet to be evaluated in the UK.

Universal (rather than targeted towards at-risk groups) early intervention programmes for school-aged children have been conducted in school settings, where the intervention is applied at the school level by either trained health professionals or school staff. One such programme ("Friends") was recently evaluated in the UK in a large ( $n=1257)$ multi-school study and the 'Friends' programme was associated with a reduction in overall anxiety scores compared to usual school provision. However, scores only reduced significantly for those children who had lower anxiety scores at baseline and when health professionals (not school staff) delivered the programme, and group differences were not maintained at a two- 
year follow-up. Such interventions are also problematic as they are resource intensive and may be financially prohibitive for schools.

\section{Special populations}

\section{Long-term physical health conditions}

Children and young people suffering from chronic physical illness have up to four times the rate of psychiatric disorder compared to those without. Rates for anxiety disorders specifically are similar, for example up to a third of young people with asthma, $13-48 \%$ of children with epilepsy, $39 \%$ of young people with Crohn's disease, and around $50 \%$ of adolescents with cystic fibrosis meet criteria for an anxiety disorder. Notably, children and adolescents with comorbid anxiety and physical health conditions have greater functional impairment than would be observed with either disorder alone. Clinicians typically perceive children and adolescents with physical and mental health conditions as complex, with the management of their physical health often taking priority. Management using medication is often not considered appropriate as trials typically exclude young people with long-term physical health conditions and there is a greater risk of adverse medication effects and drug interactions in these children. Interventions that have been evaluated in this group have been based on CBT protocols adapted from those validated for use with physically well children and young people with anxiety disorders. Adaptations to the CBT approach have been relatively minor and include a slower pace of (and therefore longer) sessions with greater emphasis on behavioural rather than cognitive aspects, extra written materials, more concrete language, and flexibility in treatment location e.g. use of telephone sessions or sessions at home, and have not usually involved specialist knowledge about the 
illness. Overall, these treatments have resulted in positive outcomes, and whilst larger RCTs are needed, the findings to date suggest that it is possible to use existing evidence-based CBT programmes to effectively treat anxiety disorders in children and young people with long-term physical illnesses.

\section{Autism Spectrum Disorders (ASD)}

Anxiety disorders are one of the most commonly occurring psychiatric conditions in young people with ASD, with recent studies indicating that approximately $40 \%$ of children and adolescents with ASD meet criteria for at least one anxiety disorder. The increased risk for anxiety in ASD is poorly understood. Furthermore, the measurement of anxiety in this population is complicated by the overlapping symptoms of anxiety and ASD e.g. social avoidance and rigidity. As such, it is unclear how best to unravel what aspects of anxiety are part of the ASD profile (e.g. anxiety related to transitions) and what reflect a separate anxiety disorder. Child self-report in this population may also be compromised by difficulty in labelling and recognising internal emotional states, and therefore multi-informant approaches to assessment are critical.

There is very little evidence regarding the effectiveness of pharmacotherapy in young people with ASD, however studies have shown that there is an increased risk for behavioural activation from SSRIs in this group. Medication is therefore not recommended as the first-line treatment for anxiety disorders in children and adolescents with ASD, and side effects should be closely monitored if used. Systematic reviews have highlighted that few psychological treatments have been developed specifically for anxiety disorders in young people with ASD. Interventions 
that have been evaluated are mainly based on modified CBT programmes and conducted in samples of young people with high functioning ASD. Encouragingly, treatment outcomes in this group are similar to those in typically developing children, with $71.4 \%$ of children recovering from their primary anxiety diagnosis following treatment.

\section{Summary and conclusions}

Anxiety disorders are common in childhood and adolescence, run a chronic course, are comorbid with other serious mental health difficulties and have a significant and enduring impact on functioning. Prompt recognition, assessment and evidencebased treatment are therefore crucial. Anxiety disorders are particularly high in children and adolescents with long-term physical health conditions and those with autism spectrum disorders. GPs and Paediatricians are well placed to identify anxiety difficulties, and refer onwards for appropriate management. Anxiety disorders can be treated effectively by CBT and/or medication (SSRIs), although CBT is recommended as the first-line treatment due to it being associated with fewer safety concerns than SSRIs, as well as being the preferred treatment among parents. Low-intensity approaches to CBT such as therapist-supported bibliotherapy and online treatments have the potential to increase access to evidence-based treatments and are likely to be cost-effective.

\section{Practice Points}

- Anxiety disorders are among the most common mental health disorders in childhood and adolescence and have a significant and enduring impact on social, academic and family functioning. 
- There are established questionnaire measures that can be used to assess symptoms of anxiety disorders in primary case, although a brief screening tool has yet to be developed. Referrals should be made to CAMHS for further specialist assessment.

- Cognitive behavioural therapy (CBT) is the first line treatment. This can be delivered in a range of ways and by a variety of trained professionals. Although CBT is effective, most children do not access treatment.

- Anxiety disorders are particularly prevalent in children and adolescents with chronic physical health problems and young people with autism spectrum disorders (ASD). However, there is some evidence to suggest generic treatments can be successfully applied to these populations.

\section{Further Reading}

Bennett, S., Shafran, R., Coughtrey, A., Walker, S., \& Heyman, I. (2015). Psychological interventions for mental health disorders in children with chronic physical illness: a systematic review. Archives of Disease in Childhood, 100(4), 308-316.

Creswell, C., Waite, P., \& Cooper, P. J. (2014). Assessment and management of anxiety disorders in children and adolescents. Archives of Disease in Childhood, 99(7), 674-678. doi:10.1136/archdischild-2013-303768

Eapen, V., \& Črnčec, R. (2014). DSM 5 and child psychiatric disorders: What is new? What has changed? Asian Journal of Psychiatry, 11, 114-118.

Ipser, J. C., Stein, D. J., Hawkridge, S., \& Hoppe, L. (2009). Pharmacotherapy for anxiety disorders in children and adolescents. Cochrane Database Systematic Review, 3(3). 
James, A. C., James, G., Cowdrey, F. A., Soler, A., \& Choke, A. (2013). Cognitive behavioural therapy for anxiety disorders in children and adolescents. The Cochrane Library.

Murray, L., Creswell, C., \& Cooper, P. J. (2009). The development of anxiety disorders in childhood: an integrative review. Psychological Medicine, 39(09), 1413-1423.

Pennant, M. E., Loucas, C. E., Whittington, C., Creswell, C., Fonagy, P., Fuggle, P., . .. Kendall, T. (2015). Computerised therapies for anxiety and depression in children and young people: A systematic review and meta-analysis. Behaviour Research and Therapy, 67, 1-18.

Rapee, R. M. (2015). Nature and psychological management of anxiety disorders in youth. Journal of Paediatrics and Child Health, 51(3), 280-284.

Sayal, K., \& Taylor, E. (2004). Detection of child mental health disorders by general practitioners. British Journal of General Practice, 54(502), 348-352.

Vasa, R. A., \& Mazurek, M. O. (2015). An update on anxiety in youth with autism spectrum disorders. Current Opinion in Psychiatry, 28(2), 83-90. 
Table 1. Common anxiety disorders in childhood and adolescence

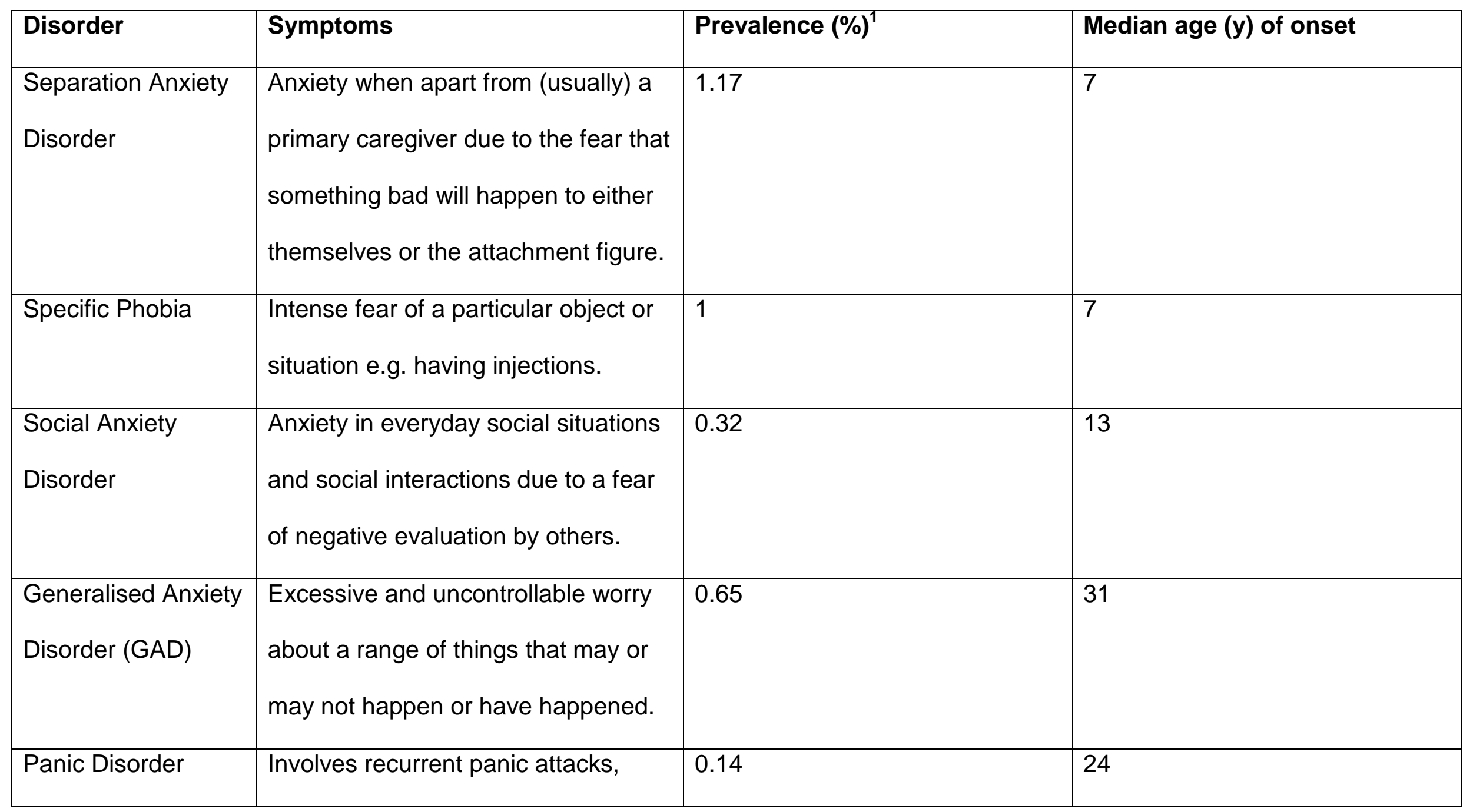




\begin{tabular}{|c|c|c|c|}
\hline & $\begin{array}{l}\text { which are feelings of terror that occur } \\
\text { suddenly and without warning, } \\
\text { accompanied by physical symptoms } \\
\text { such as chest pain, palpitations, } \\
\text { sweating and feeling of choking. }\end{array}$ & & \\
\hline Agoraphobia & $\begin{array}{l}\text { The fear of actual and anticipated } \\
\text { places or situations that might result } \\
\text { in a panic attack and often involves a } \\
\text { belief that there is no easy way to } \\
\text { escape or seek help. }\end{array}$ & 0.07 & 20 \\
\hline
\end{tabular}

${ }^{1}$ Figures represent point prevalence (proportion who meet criteria for a diagnosis at a specific point in time)

${ }^{2}$ This figure is taken from a different study to the other disorders. It is higher than might be expected, possibly due to methodological differences between the studies. 